\title{
Automation of Data Traffic Control on DSM Architectures
}

\author{
Michael Frumkin, Haoqiang Jin, and Jerry Yan ${ }^{\star}$ \\ Numerical Aerospace Simulation Systems Division \\ NASA Ames Research Center
}

\begin{abstract}
The distributed shared memory (DSM) architecture simplifies development of parallel programs by relieving a user from the tedious task of distributing data across processors. Furthermore, it allows incremental parallelization using, for example, OpenMP or Java threads. While it is easy to demonstrate good performance on a few processors, achieving good scalability still requires a good understanding of data flow in the application. In this paper we discuss ADAPT, an Automatic Data Alignment and Placement Tool, that detects data congestions in FORTRAN array oriented codes and suggests code transformations to resolve them. We then show how ADAPT suggested transformations, including data blocking, data placement, data transposition and page size control improve performance of the NAS Parallel Benchmarks.
\end{abstract}

\section{Significance of Data Traffic Control}

Few programming constructs allow explicit specification of data location in computer memory 1 . That leaves data placement decisions to the compiler and the operating system. The time for accessing data varies across different computer architectures, making code tuning machine dependent. Some memory problems such as cache trashing and false sharing are not difficult to identify and overcome with array dimension padding and variable privatization. Other data traffic problems such as data locality, excessive TLB misses and thread interference are difficult to diagnose and cure. Even the best implementations of Computational Fluid Dynamics (CFD) codes can achieve only about $20 \%$ of machine peak performance 14, spending $80 \%$ of the time waiting for data.

Two main factors contribute to the low efficiency of these codes. First, the distribution of Floating Point Instructions (FPI) does not present an optimal mix to keep all the functional units busy. There is no obvious cure for this. Second, many operations are stalled waiting for data, see Example 1 in Section 2. Solving this problem is the subject of the paper.

Approach to data traffic optimization. The first step in addressing this challenge is to identify code constructs causing data congestion, and the type of

* M/S T27A-2, NASA Ames Research Center, Moffett Field, CA 94035-1000; e-mail: \{rumkin, hjin, yan\}@nas.nasa.gov

${ }^{1}$ By explicit constructs, we mean statements such as the "register" qualifier in $\mathrm{C}$ or data distribution directives in High Performance Fortran. 
congestion. We use four congestion metrics in this paper: Primary Data Cache (PDC) misses, Secondary Cache (SC) misses, Translation Lookaside Buffer (TLB) misses, and Cache Invalidations (CI). The second step involves choosing a proper remedy to resolve congestions and applying it to the code. This remedy can be either code transformation, or changing program environment such as page size or page placement/migration mechanisms.

Methods for controlling data traffic. These problems have been studied elsewhere [410:11] and techniques have been developed to improve data traffic. These techniques include data grouping for cache optimization, optimization of the page size for reduction of TLB misses, placement of pages to resolve contention in memory channel and data transposition for reduction of cache invalidations and TLB misses. Even with these techniques in hand, it is not easy for the user to identify the appropriate transformations to be applied to various part of the code. For example, it has been known that computations of the rhsz and zsolve operators in BT and SP of the NAS Parallel Benchmarks (OpenMP version) exhibit performance which is 3-4 times worse than of corresponding operators in the $x$-direction [7]. Neither, the reason for this, nor a mechanism for fixing the problem has been known in spite of NPB being de facto standards for reporting improvements in compilers and tools [12].

Many code transformations to improve data traffic are already implemented in compilers. These include loop interchange, loop fusion, software pipelining, prefetching, and modification of variable declarations (such as padding and privatization). Nevertheless, compilers cannot perform expensive analysis involving full interprocedural data dependency analysis. Many types of analysis are not possible at compile time at all.

Another approach to identifying data traffic problems relies on hardware counters that gather event statistics during program execution. Tools for collecting and analyzing the events, such as perfex and Performeter, have been built on top of these counters $[613$. These tools allow a user to instrument code and identify code constructs with performance anomalies. However, these tools are diagnostic in nature and leave analysis of the problems and identification of the remedies to the user.

In this paper we present ADAPT, a tool that combines both static code analysis and run-time testing. ADAPT analyzes data-to-data and data-to-computations affinity in the source code to report potential data traffic problems. The tool also inserts diagnostic statements in the code for those expressions that influence data traffic but cannot be evaluated at compile time. These statements are evaluated at run time and issue warnings about poorly performing code constructs. We demonstrate ADAPT's ability to identify problematic data traffic constructs and to suggest cures for these problems on three simulated CFD applications BT, SP and LU of the NAS Parallel Benchmarks (NPB). ADAPT was able to resolve data traffic problems with the rhsz and zsolve operators and helped to improve performance of the codes by $27 \%$ on average. 


\section{Automated Detection of Data Traffic Problems}

To be able to control data traffic in an application, the user needs information on data movement across the memory hierarchy. While details of such movement can be complicated and machine dependent, they can be formulated in terms of cache parameters, array offsets, and data access strides, and can be characterized by simple metrics such as cache misses and invalidations. In a few cases, such as accessing shared data, data traffic depends on the cache coherency protocol and is sensitive to variations in execution order across different threads.

A tool that detects and corrects data traffic congestions greatly reduces the burden on a user to tune his code manually. Such a tool can reduce data traffic by increasing data reuse, avoid memory contention by optimizing initial data placement, and reduce the number of TLB misses and thread interference by optimizing page size. A typical scenario in which such a tool is applied is shown in the following example.

Example 1. The first two loop nests in lhsz of SP from NPB (serial version) are shown in Figure 1, left pane. The attempt to optimize the first nested loop actually slows down the computations since it increases the number of accessed memory pages and poorly utilizes the primary cache, see lhsz curve in Figure 2 Merging the first and the second nested loops and recalculating the expressions, see right pane in Figure 11 decreases the number of pages accessed, improves cache utilization, and reduces the total execution time, in spite of increasing the total number of FPI, see lhsz_t curve in Figure 2,

We have implemented such a tool by adding features to ADAPT (Automatic Data Alignment and Placement Tool) 2]. Originally, ADAPT was designed for the automatic insertion of HPF directives into FORTRAN code2. The tool is able to identify data-to-data and to data-to-computations affinity and to express such affinities through HPF ALIGN and DISTRIBUTE directives. ADAPT's ability to extract these affinities is the key to enabling it with automatic data traffic diagnostic capabilities.

Data-to-data affinity. Two data items have an affinity if both are required by the same instruction (directly or indirectly) during program execution. For a pair of arrays used in the same loop nest statement, the affinity relation is a correspondence between array elements referenced by the same loop index. Grouping of affine data items and packing the groups into a continuous stream improves program performance by hiding the memory latency. In general, affinity is a many-to-many relation leaving many degrees of freedom to group affine data items. In [3], it is shown that the efficiency of grouping affine array elements depends on the geometry of the array interference lattice, defined as a set of solutions to the Cache Miss Equation [4].

The affinity relation can be deduced for each array pair in each nest statement. A control dependence results in affinity relations between the arrays involved in the control statement and all arrays in the basic blocks immediately

${ }^{2}$ ADAPT is built on top of CAPTools [9. It uses a CAPTools generated data base and CAPTools code analysis and utilities. 


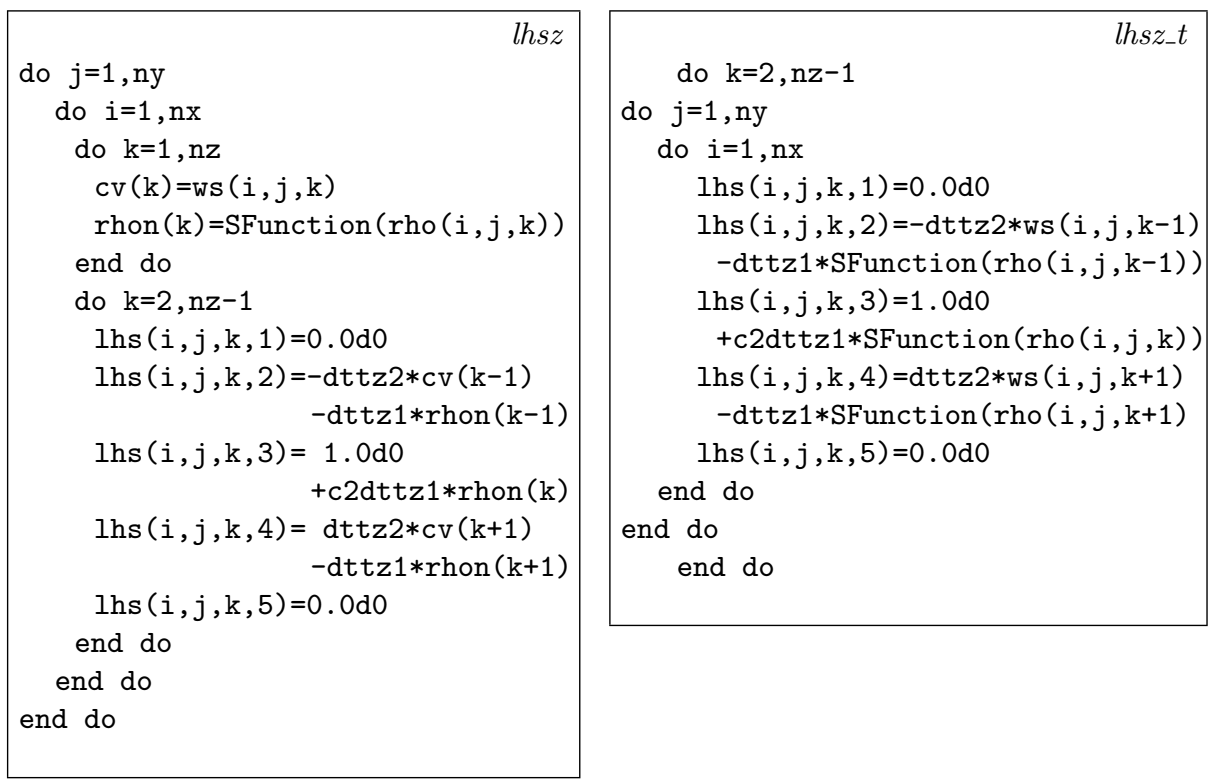

Fig. 1. Data Traffic Optimization. The original code (left), taken from 1hsz.f of NPB2.3-serial, saves floating point instructions used in SFunction. However, such loop ordering creates a large number of TLB and PDC misses. By rearranging computations (right pane) these problems are resolved, improving execution time in spite of increase in the number of FPI. The profiles of both codes are shown in Figure 2 .

dominated by the statement. The most common case observed in our CFD applications is one-to-many affinity relations between arrays resulting from difference operators on structured discretization grids. These relations can be described by a stencil (i.e. by a set of vectors with constant elements) and we call them stencil relations. In order to deduce the affinity for arrays used in different statements of the same nest, ADAPT uses the chain rule, see [2]. The union of affinity relations over all directed paths leading from an array $u$ to an array $q$ forms the nest affinity relation between $q$ and $u$. The relation lists all elements of $u$ used for computation of each element of $q$ and is a one-to-many mapping.

Data-to-computations affinity. We represent a program by a bipartite graph called the program affinity graph. Let $C$ be the set of program statements, and let $D$ be the program data, i.e. the set of (virtual) memory locations referenced in the program. We say a memory location $d$ has an affinity with a statement $c$ if the datum at address $d$ is either an operand or a result of $c$. The program affinity graph has $C$ and $D$ as the vertices of the parts with appropriately directed arcs connecting statements with data affine to them. Many program properties can be expressed in terms of the affinity graph. For example, a statement $c 2$ depends on a statement $c 1$ if there is a directed path from $c 1$ to $c 2$. Otherwise, $c 1$ and $c 2$ are independent and can be executed in any order.

The analysis of the affinity graph can be simplified by indexing the statements inside the nests and the memory locations used by the arrays. In this 


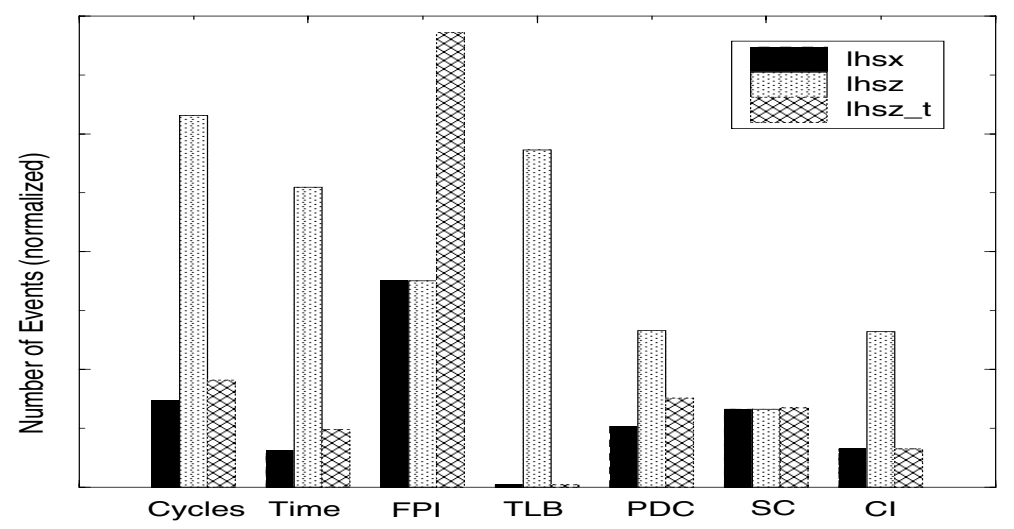

Fig. 2. The effect of TLB (Table Lookaside Buffer) and PDC (Primary Data Cache) usage optimization on the performance of lhsz nest. The performance of a similar operator in $x$-direction (lhsx) is given as a reference. The horizontal axis shows types of events measured with the use of hardware counters. The vertical axis shows a normalized number of measured events. As above, FPI stands for Floating Point Instructions, $\mathrm{SC}$ for Secondary Cache misses, and CI for secondary Cache Invalidations.

case, the arcs connecting data and statements can be expressed as $(I ; \operatorname{idx}(I))$ where $I$ is a loop nest index tuple, and $\operatorname{idx}(I)$ is a memory address of an array element referenced in iteration $I$. In our application domain (CFD applications on structured grids), the index function is usually a linear function of $I$ with symbolic coefficients known at compile time. There are only a few nests in our applications where this is not a case. These include the core of the FFT algorithm, where $\operatorname{idx}(i, j, k)=i+2 \cdot j \cdot k$; nests interpolating data between distinct grids, where idx function is read from a file; nests accessing specially enumerated grid points where the idx function is stored in a precomputed array. The tool identifies nests with nonlinear access functions, without any further analysis.

Some properties of the data traffic can be deduced using only symbolic analysis of the idx function (see the thread noninterference condition below). Others require knowledge of the actual numerical values of the coefficients. If the properties of the data traffic can be expressed by a symbolic expression but cannot be verified without knowing the numerical values of the coefficients, ADAPT inserts the expression in the code and the user receives a warning at run time. We call this a run time test.

Checking cache unfriendly access patterns. In general, cache friendly computations involve good temporal and spatial locality [5] and cannot be expressed in simple closed-form terms 4 . However, some necessary conditions for cache friendly computations can be formulated and checked. The first condition is simple: array elements accessed in the iterations of the innermost loop cover contiguous memory locations. Otherwise, nonunit stride in memory access would cause underutilization of data loaded into the cache. 
Detection of self interference. If an array self affinity relation is a stencil relation, ADAPT represents the addresses of the corresponding array elements as a polylinear function of array sizes and the index coefficients. For each possible pair of the stencil vectors, ADAPT generates a set of constraints for the array dimensions of the form $n x \cdot n y \neq m / h \cdot S$, where $n x, n y$ are the array sizes, $S$ is the primary cache size, $m$ is any positive integer, and $h$ is an empirically chosen small integer $(h=1,2, \mathrm{cf}$. [3]). If neither $n x$ nor $n y$ is known at compile time, a satisfiability test for these constraints is inserted into the program as a run time test.

Detection of cross interference. Cross interference between two arrays happens when affine elements are mapped to the same cache location. Detecting cross interference is similar to identifying self interference, except that it involves the inter-array offset and the dimensions of both arrays. The cross interference constraints are represented by a set of polylinear inequalities: $\operatorname{idx}_{a}\left(i+e_{1}, j+e_{2}, k+e_{3}\right)+\operatorname{off}_{a b}-\operatorname{idx}_{b}\left(i+d_{1}, j+d_{2}, k+d_{3}\right) \neq m / h \cdot S$, where $e_{i}, d_{i}, i=1,2,3$ are components of the stencil vectors. Evaluation of these inequalities requires knowledge of off $a b$ and can be done, for example, if both arrays are in the same common block or are segments of the same (bigger) array.

Detection of high TLB misses. TLB misses (as in Example 1) usually result from large memory strides due to iterations of an inner loop of a nest. For each array in the nest our TLB miss test checks a sufficient conditions for TLB misses in the inner loop: the number of iterations of the innermost nest exceeds TLB_SIZE, and the distance between the first and last address accessed in the inner loop exceeds PAGE_SIZE*TLB_SIZE. If both conditions are met, ADAPT issues a warning about high TLB misses in the inner loop of the nest. If both conditions can not be proved to be false, the tool inserts a run time test.

Checking thread noninterference. This condition can be formulated as absence of overlap of the address spaces accessed by different threads. If the noninterference condition is satisfied, then the memory accessed by a thread can be placed in the memory of the processor running the thread, improving data locality. This condition is checked only for "read/write" arrays since thread interference would cause cache lines invalidations. In the case of "read" arrays, this condition is not checked since read arrays are copied into secondary cache and threads do not affect each other, provided that initial placement of the array has been done correctly.

Interference of threads depends on the data sharing protocol implemented in the DSM computer. For example, if data coherence is supported at the level of secondary cache lines, as it is on the Origin 2000, then read/write interference can happen if two threads are accessing two different words on the same cache line. For a software performance tool, it is possible to be aware of the data sharing protocol and adjust CI estimates accordingly. We have implemented a more general approach: for each nest and each thread, ADAPT evaluates the interference as a ratio of the number of memory locations adjacent to the memory locations accessed by other threads to the total number of memory locations 
accessed by the thread. For example, in Figure1 the interference ratio for 1 hs is $P /(n x \cdot n y)$ in lhsz and $P /(n x \cdot n y \cdot n z)$ in lhsz_t, which correlates well with the $\mathrm{CI}$ numbers in Figure 2. This interference indicator is similar to the surface-tovolume ratio used to estimate cache utilization [3], and to the communicationsto-computations ratio used to characterize MPI programs.

Detection of the data sources and the initial data placement. Page placement on a DSM computer is commonly controlled by a simple policy such as "First Touch" or "Round Robin". More sophisticated page placements can be implemented with special tools, such as dplace, see [13]. An inappropriate initial data placement - for example, concentrating data on a single processor - can cause memory contention during execution and may hamper application scalability. Therefore, we enabled ADAPT with a capability to detect data initialization constructs in the code and to insert an equitable data placement directive before each construct.

\section{Experiments}

We conducted experiments on 16 processors of a 512 processor single image SGI Origin 2000 (MIPS R12000 CPU). We submitted jobs through the Portable Batch System (PBS), which dedicates requested resources to the job and minimizes interference with other jobs running on the machine. We used 16 processors, since this is the minimal number of processors where the slowdown due to memory traffic effects was well pronounced for the $64^{3}$-point grids of NPB class A. The effects are similar up to 32 processors, after which the load imbalance becomes dominant.

The primary memory hierarchy on the Origin 2000 involves registers, primary data and instruction caches, secondary unified data and instruction cache, and the main memory. The access time to data located at different levels of memory is shown in Table 1 , cf. [13]. The primary cache is directly mapped 2-way set

Table 1. Access Time (in machine cycles) to Data in Origin 2000 Memory.

\begin{tabular}{|c|c|l|}
\hline \hline Data Location & Access Time & Condition \\
\hline \hline registers & 0 & \\
\hline L1 cache & $2-3$ & L1 hit \\
\hline L1 cache & $8-10$ & L1 miss, L2 hit \\
\hline L2 cache & $75-250$ & L2 miss, TLB hit \\
\hline L2 cache & $\sim 2000$ & L2 miss, TLB miss \\
\hline
\end{tabular}

associative, having 512 lines of 32 bytes each in each set. The secondary cache is shared by data and instructions and it is also directly mapped 2-way set associative containing $32 \mathrm{~K} 128$ byte lines. The main memory is split in modules of $768 \mathrm{MB}$ per node (2 processors per node), totaling 196 GB of memory on a 512 processor machine. The TLB has 64 entries containing base addresses of 64 pairs of pages. 
A cache coherency protocol guarantees that data accessed by different processors do not go stale. This protocol invalidates a line in secondary cache every time a processor requests exclusive ownership (usually for writing) of data mapped to the line. In this case, all copies cached in all other processors are invalidated, and each processor working with this line has to request a fresh copy of the line to resume computations. An implication of this protocol is that it will cause significant slowdown when two processors attempt to write data located within the same 128 byte segment of main memory.

As the test codes, we chose the OpenMP version of the PBN3.0-b2, a release of the NAS Parallel Benchmarks which includes optimized serial, OpenMP, HPF and Java versions. The suite is designed for demonstrating capabilities of compilers and tools applied to CFD codes [7].

We measured execution time at various levels of optimization. Since we used the -03 flag in the compilation, special effort was required in many cases to prevent the compiler from undoing our optimizations. We specified compiler flags for suppressing prefetching: --LNO: prefetch $=0$ to obtain accurate numbers for the hardware counters, and flag OPTFLAGS=-OPT: reorg_common=0FF to enforce our own padding of arrays declared in the common blocks.

\section{Experimental Results and Discussion}

We applied the tool to the SP, BT and LU codes of the NPB [7] (optimized OpenMP version PBN-O). For each code ADAPT was able to generate the following data traffic optimization diagnostics.

- Nests for initial data placements. ADAPT detected all nests where data were initialized. In all cases, the arrays were initialized from array of smaller dimensions (or constants). The initial data placement was appropriately implemented in the original code and we essentially did not make any changes.

- Nests with nonunit strides and advice on loop interchange. Such nests were detected only in calculation of the so-called right hand side array by the subroutines rhs, exact_rhs, and erhs.

- Nests with big strides and advise on loop interchange and data transpositions. Nests with big strides were detected in the subroutines rhs, exact_rhs, erhs and in zsolve. Advice on changing $j i k$ into $k j i$ loops was issued for the first three subroutines. In zsolve (BT and SP only), a dependency in the $k$ index prevented the parallelization of the $k$ loop, and no loop interchange was carried out.

- Nests with self or cross interference, and advice on padding. No arrays with self interference were detected indicating that the existing paddings of the first and second dimensions were sufficient. The cross interference condition was presented in the form that array offsets cannot be equal to a multiple of the cache size plus a stencil vector offset.

Following ADAPT's advice, we implemented a number of changes in the original OpenMP code by hand. Almost all changes occurred in rhs, zsolve, buts, and blts. We classify these changes into 3 categories, as shown in Table 
2: removing auxiliary arrays and applying nest fusion in rhs, loop interchange in rhs, removing auxiliary arrays in solvers: zsolve in BT and SP and buts, blts in LU. Both exact_rhs and erhs were outside the main iteration loop, so we did not make any changes to these subroutines. Incremental improvement in performance via data traffic optimization for each benchmark is tabulated in Table 2. The improvement was about $200 \%$ for rhs and $20 \%$ for zsolve. Average overall speedup was about $27 \%$ on 16 processors. Some optimizations that gave

Table 2. Improving Benchmark Performance via Data Traffic Optimization. Time (in seconds) was measured on 16 processors of a 400MHz Origin 2000 machine, SGI OpenMP compiler.

\begin{tabular}{|c|c|c|c|c|c|}
\hline \hline $\begin{array}{c}\text { Appli- } \\
\text { cation }\end{array}$ & $\begin{array}{c}\text { Original } \\
\text { Code }\end{array}$ & $\begin{array}{c}\text { Data reuse } \\
\text { and nest fuse }\end{array}$ & $\begin{array}{c}\text { Loop } \\
\text { interchange }\end{array}$ & $\begin{array}{c}\text { Removing solver } \\
\text { aux. arrays }\end{array}$ & $\begin{array}{c}\text { Total } \\
\text { speedup }\end{array}$ \\
\hline \hline BT.A & 54.00 & 51.44 & 44.22 & 42.12 & $22 \%$ \\
\hline SP.A & 63.40 & 55.89 & 38.70 & 37.92 & $40 \%$ \\
\hline LU.A & 59.35 & 52.19 & 48.95 & 48.78 & $18 \%$ \\
\hline
\end{tabular}

performance improvements in simple test codes did not result in expected improvements in the benchmarks. Optimizing page size by providing each processor with one page from each array did not affect performance of the jobs running under PBS. It improved performance of jobs running outside PBS by $10 \%$. We could not find a remedy for the thread interference warning (high CI number) in zsolve, because the nest has a dependency in the $z$-direction, preventing loop interchange.

\section{Conclusions and Related Work}

In this paper we presented ADAPT, a tool to identify data traffic problems in array-oriented Fortran codes. The tool advises the user on excessive cache and TLB misses, and possible thread interference, and suggests code transformations for improving data traffic. Some of the transformations are counterintuitive, since they reduce data traffic and overall computational time by increasing the number floating point instructions.

We demonstrated data traffic improvements using three simulated CFD applications BT, SP and LU from the NAS Parallel Benchmark suite. For some subroutines performing operations in the $z$-direction, code transformations improved performance by a factor of 3 and improved scalability of these subroutines. Overall data traffic optimizations improved the benchmark performance on 16 processors by $27 \%$ on average.

Research is being carried out in three main directions to enhance ADAPT's ability to control data traffic: reducing communications in MPI programs [9], optimizing data distributions in HPF programs [2], and improving spatial and 
temporal data locality for optimizing cache performance [4]. With the proliferation of the DSM architecture, data traffic control on DSM is becoming increasingly important. Some problems of data distributions and page migrations on DSM are the subject of recent papers 10[11. We have plans to implement some global data traffic control features in ADAPT and to test it on a wider class of CFD applications and on variety of different implementations of DSM. Finally, we plan to incorporate ADAPT with CAPO, a parallelization tool that automatically inserts OpenMP directives into FORTRAN codes 8 .

Acknowledgements. This work was supported by the NASA High Performance Computing and Communications (HPCC) Program, RTOP \#725-10-31. The authors appreciate Rob F. Van Der Wijngaart's effort in reviewing this paper.

\section{References}

1. D. Bailey, J. Barton, T. Lasinski, and H. Simon (Eds.). The NAS Parallel Benchmarks. NAS Technical Report RNR-91-002, www.nas.nasa.gov.

2. M. Frumkin, J. Yan. Automatic Data Distribution for CFD Applications on Structured Grids. The 3rd Annual HPF User Group Meeting, Redondo Beach, CA, August 1-2, 1999, 5 pp. Full version: NAS Technical report NAS-99-012, December 99. $27 \mathrm{pp}$.

3. M. Frumkin, R.F. Van der Wijngaart. Efficient Cache Use for Stencil Operations on Structured Discretization Grids. Submitted to JACM, see also NAS Technical Report NAS-00-15, www.nas.nasa.gov.

4. S. Gosh, M. Martonosi, S. Malik. Cache Miss Equations: An Analytical Representation of Cache Misses. ACM ICS 1997, pp. 317-324.

5. J.L. Hennessy, D.A. Patterson. Computer Organization and Design. Morgan Kaufmann Publishers, San Mateo, CA, 1994.

6. Innovative Computing Lab., UTK. PAPI: Hardware Performance Counter Application Programming Interface. http://icl.cs.utk.edu/papi.

7. H. Jin, M. Frumkin, J. Yan. The OpenMP Implementation of NAS Parallel Benchmarks and Its Performance, NAS Technical Report, NAS-99-011, 1999, 26 pp, www.nas.nasa.gov.

8. H. Jin, M. Frumkin, J. Yan. Automatic Generation of OpenMP Directives and Its Application to Computational Fluid Dynamics Codes, Springer LNCS, v. 1940, p. $440-456$.

9. S.P. Johnson, C.S. Ierotheou, M. Cross. Automatic Parallel Code Generation on Distributed Memory Systems. Parallel Computing, V. 22 (1996), pp. 227-258.

10. D. S. Nikolopoulos, T. S. Papatheodorou, C. D. Polychronopoulos, J. Labarta, E. Ayguade. Is Data Distribution Necessary in OpenMP? Proceedings of Supercomputing 2000. Dallas, TX, Nov. 4-10, 2000. 14 pp.

11. D. S. Nikolopoulos, T. S. Papatheodorou, C. D. Polychronopoulos, J. Labarta, E. Ayguade. Leveraging Transparent Data Distribution in OpenMP via User-Level Dynamic Page Migration. Springer LNCS, v. 1940, p. 415-427.

12. Proceedings of Supercomputing 2000. Dallas, TX, Nov. 4-10, 2000.

13. SGI Inc. Technical Document. Origin2000 and Onyx2 Performance Tuning and Optimization Guide. http://techpubs.sgi.com.

14. J. Taft. Performance of the Overflow-MLP CFD Code on the NASA Ames 512 $C P U$ Origin System. NAS Technical Report, NAS-00-05. www.nas.nasa.gov. 